\title{
RSS based Cell Fingerprint Patterns and Algorithms for Cell Identification in the Context of Self-organized Energy Saving
}

\author{
Elke Roth-Mandutz \\ IImenau University of Technology \\ IImenau \\ Germany \\ elke.roth-mandutz@tu- \\ ilmenau.de
}

\author{
Stephen S. Mwanje \\ IImenau University of Technology \\ IImenau \\ Germany \\ stephen.mwanje@tu- \\ ilmenau.de
}

\author{
Andreas Mitschele-Thiel \\ IImenau University of Technology \\ IImenau \\ Germany \\ mitsch@tu-ilmenau.de
}

\begin{abstract}
Due to the large number of newly deployed small cells in heterogeneous networks needed for off-loading the mobile data traffic, the energy consumption in mobile cellular networks is strongly increasing. Energy consumption can be optimized in a self-organized way by adapting the number of active cells to the current traffic demand. In this paper we concentrate on how to identify small cells to be reactivated. Solely based on the received signal strength, we present cell-specific patterns for the generation of unique cell fingerprints. Using matching algorithms, the cell fingerprints of the deactivated cells are matched with a measurement sample from a User Entity (UE) to identify the most appropriate candidate. Our results give a matching success rate of $95 \%$ to identify the best cell to be activated.
\end{abstract}

\section{Categories and Subject Descriptors}

G.3 [Probability and Statistics]: Probabilistic algorithms; I.5 [Pattern Recognition]: Statistical

\section{General Terms}

Algorithms, Measurement, Design, Verification.

\section{Keywords}

Fingerprinting, energy saving SON, LTE, small cells

\section{INTRODUCTION}

The continuously massive global mobile data growth demands the deployment of a large number of additional base stations. For offloading the mobile data traffic, legacy networks are expanded by

Permission to make digital or hard copies of all or part of this work for personal or classroom use is granted without fee provided that copies are not made or distributed for profit or commercial advantage and that copies bear this notice and the full citation on the first page. To copy otherwise, to republish, to post on servers or to redistribute to lists, requires prior specific permission and/or a fee.

MOBIQUITOUS 2014, December 02-05, London, Great Britain Copyright $\odot 2014$ ICST 978-1-63190-039-6

DOI 10.4108/icst.mobiquitous.2014.258231 multiple overlaying small cells causing an energy consumption rise for cellular networks of $40 \%$ from 2010 to 2020 [2]. Therefore, environmental and economic reasons require the introduction of efficient energy saving approaches without impacting the network quality.

As cellular networks are generally dimensioned for peak traffic hours, energy can be saved using the energy saving SelfOrganized Networks (SON) use case [1] by adjusting the network capacity to the current traffic demand. Considering the strong local and periodical mobile traffic variations during the course of the day [2], small cells of a heterogeneous network may be deactivated during low traffic periods and reactivated on a need basis. The challenge of the energy saving SON is how to identify the relevant cell(s) to be reactivated in time to satisfy the upcoming traffic demand. In this paper we present the fingerprinting method for cell identification based on cell patterns used for cell fingerprint generation and corresponding matching algorithms.

\section{FRAMEWORK FOR CELL FINGERPRINT ANALYSIS AND MATCHING}

Our cell fingerprinting system involves two phases as detailed in [4]: an initial learning phase followed by the operational phase. During the learning phase the cell fingerprints are generated and stored in the reference database. In the operational phase the cell initiates the cell identification and activation procedure, when the traffic in an active cell exceeds a predefined cell load. UE measurements are retrieved and matched against the cell fingerprints of all deactivated cells stored in the reference database of the highly loaded cell. The best matching cell is identified as the cell providing the best radio conditions to take over traffic from the overloaded cell.

The cell fingerprints are solely based on the neighbor cell received signal strength (RSS) as measured by the UE. Reliable cell fingerprints demand RSS measurement samples to be captured from multiple UEs at various positions within each cell. Figure 1 shows three exemplary RSS distributions of an intra-Base Station (BS), an adjacent inter-BS cell and a non-adjacent neighbor cell. Based on the analysis of multiple RSS distributions, the following RSS distribution characteristics were found: 


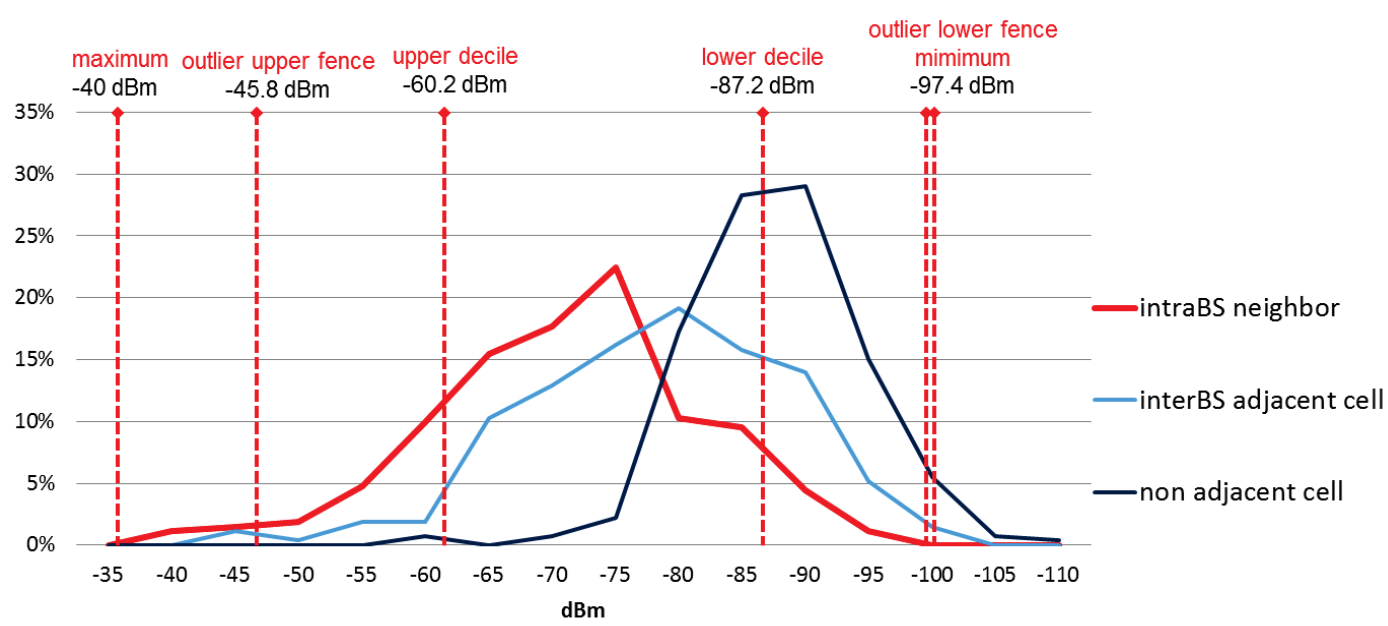

Figure 1. RSS distributions of 3 different types of neighbor cells superimposed with the cell patterns for the intraBS neighbor cell

\begin{tabular}{|c|c|c|c|c|c|c|}
\hline \multicolumn{7}{|c|}{ fingerprint(cell $I_{0}$ ) } \\
\hline $\begin{array}{c}\text { rss patterns } \vec{\longrightarrow} \\
\text { neighbor cells } \boldsymbol{\nabla}\end{array}$ & $\begin{array}{l}\text { upper } \\
\text { minMax }\end{array}$ & $\begin{array}{l}\text { lower } \\
\text { minMax }\end{array}$ & $\begin{array}{c}\text { upper } \\
\text { exclOutlier }\end{array}$ & $\begin{array}{c}\text { lower } \\
\text { exclOutlier }\end{array}$ & $\begin{array}{l}\text { upper } \\
\text { decile }\end{array}$ & $\begin{array}{l}\text { lower } \\
\text { decile }\end{array}$ \\
\hline cell ID & & & & & & \\
\hline cell ID $D_{2}$ & & & & & & \\
\hline$\ldots$ & & & & & & \\
\hline cell $I D_{x}$ & & & & & & \\
\hline
\end{tabular}

Figure 2. Cell fingerprint

- Caused by the distance to the neighbor cell and environmental conditions, the RSS neighbor cell distributions have different minimum and maximum RSS values.

- Due to common radio effects, such as shadowing, reflection and interference, the measurement distributions for neighbor cells do not completely correspond to normal distributions. As the RSS distributions are unevenly distributed with varying ranges, quantiles are considered for modeling the RSS distributions.

- Outliers i.e. RSS values numerically distant from the rest of the RSS measures were observed. Outliers are unavoidable in practice [5] and should be treated differently to ensure robust fingerprints. For outlier calculation we use the widely used approach based on the interquartile range [5] where any measurement outside the quartile-based upper and lower fence is seen as outlier.

Resulting from these observations 3 cell patters are derived as indicated in Figure 1:

1. the absolute minimum to maximum RSS range referred to as minMax

2. the upper and lower RSS range excluding outliers referred to as exclOutlier

3. the decile range excluding the upper and lower decile referred to as decile
Each sample of fence values for a given neighbor cell ID result in one data record of the cell fingerprint. The complete cell fingerprint consists of a list of all neighbor cell IDs with a data record for each neighbor as shown in Figure 2.

To initiate the cell identification procedure as detailed in [4], a UE measurement sample is retrieved from the overloaded cell. The overloaded cell matches the retrieved UE sample with each fingerprint representing a deactivated neighbor cell. For each cell pattern a separate score is incremented, if the received UE measurement for a given neighbor cell lies within the respective range. The cell(s) with the highest number of scores are determined as the best fitting cell(s) to be activated.

\section{SIMULATION SCENARIO AND ENVIRONMENT}

Simulation studies were done using a $\mathrm{C}++$ based event-driven LTE downlink system level simulator based on libraries provided by Alcatel-Lucent Bell Labs Germany and University of Stuttgart's Institute of Communication Networks and Computer Engineering. The network environment assumes an urban area covered by a small-cell network overlaid with a legacy network. Cells in the overlay network are considered to be always on, ensuring stable coverage for low data rate users. The simulated small cell network consists of 21 cells expected to serve high data 
Table 1. LTE simulator - system parameters and values

\begin{tabular}{|l|l|}
\hline Parameter & Value \\
\hline Network size & 21 cells (7 base stations each with 3 cells) \\
\hline System bandwidth & $10 \mathrm{MHz}$ \\
\hline Simulation time step & $20 \mathrm{~s}$ \\
\hline Inter-site distance & $500 \mathrm{~m}$ \\
\hline Pathloss & $128.1+37.6 \log 10(\max (\mathrm{dKm}, 0.035))$ \\
\hline Shadowing & standard deviation $6 \mathrm{~dB}$, decorrelation distance $50 \mathrm{~m}$ \\
\hline eNB Tx power & $46 \mathrm{dBm}$ \\
\hline eNB Tx antennas & 1 per sector with max. gain $15 \mathrm{dBi}$ at $32 \mathrm{~m}$ \\
\hline UE receive antennas & 1 omni with max. gain $2 \mathrm{dBi}$ at $1.5 \mathrm{~m}$ \\
\hline Number of UEs & 3 mobile groups with 20 mobiles each \\
\hline & random walk - pedestrian: $2.4-3.6 \mathrm{~km} / \mathrm{h}$ \\
UE Mobility & - bicycle: $\quad 12-18 \mathrm{~km} / \mathrm{h}$ \\
& \multicolumn{2}{c}{$24-36 \mathrm{~km} / \mathrm{h}$} \\
\hline
\end{tabular}

rate UEs. Any of the small cells may be deactivated or activated depending on the prevailing traffic demand. Users, which are initially randomly placed in the coverage area, move with a random-walk mobility model.

The simulation is split into two phases, the cell fingerprint generation and the cell identification phase. We assume that all users are connected to the network throughout the complete simulation interval. The generation of reliable cell fingerprints requires UE measurements from different positions per cell. Therefore, three mobile user groups each with 20 users are considered. Each group uses a defined speed varying by up to $40 \%$. Further details of the simulation assumptions and parameters are given in Table 1.

For cell fingerprinting the relevant measurements are the unique identification of the neighbor cell ID and the associated RSS cell patterns. A snapshot of UE measurement samples from all UEs is taken every 20 seconds. To ensure uniformly distributed statistics for fingerprint generation, simulations are repeated for 25 resp. 40 simulation runs with $100 \mathrm{~s}$ each. The number of samples per cell ranges between 276 to 450 for 25 and 518 to 691 for 40 simulation runs with approximately $1.4 \%$ outliers. On completion the cell fingerprints are calculated. For the cell identification the last recorded measurement sample of the consecutive simulation run is used. In each simulation run one sample per cell is taken and matched against all cell fingerprints.

\section{SIMULATION RESULTS}

For the matching, we wish to determine the deactivated cell to which the candidate UE would be connected at the time the UE measurement sample was sent, if all cells had been active. We distinguish two cases indicated by the two colors in Fig. 3. The first given in dark green, indicates when the cell patterns identify exactly one cell as the best cell, while the light green is the case when the cell patterns identify not only one, but a set of cells as the best candidate. The average number of candidate cells identified by each of the patterns is given by Table 2 .

Owing to the wide range of the minMax pattern, many cells are assumed as valid neighbors, sometimes even wrongly. Resulting, $\operatorname{minMax}$ is never able to identify a single cell as the best candidate, but will always identify it among a set of cells. Consequently, the minMax pattern reaches the high hit rate of $95 \%$, if the best cell is identified as one out of multiple best cells, but never isolates that best cell.

The exclOutlier pattern improves the result on isolating the best candidate (up to $14 \%$ ), but with worse general results, when the two cases are combined (90\%). Thus, removing outliers causes significantly less false positive reducing the average number of candidates (i.e. cells with the same high score) to 3.5 as shown in Table 2.

Similar behavior is observed with the decile pattern, which improves the isolation of a single candidate to $52 \%$ albeit with a smaller general result of only $64 \%$, when both cases are combined.

Concluding, both the minMax and exclOutlier pattern provide good cell identification, if multiple cell activation and thus a negative impact on the energy saving is acceptable. The decile pattern provides a promising option to identify a single cell with the risk to activate a cell not offering the optimal radio conditions.

\section{CONCLUSION AND FUTURE OUTLOOK}

In this paper we show, that cell fingerprinting is a promising approach for the identification of inactive cells in cellular networks. Cell fingerprinting may also be applicable in other cell location sensitive applications, such as macrocell to microcell handover to identify the best cell to take over the data traffic. 


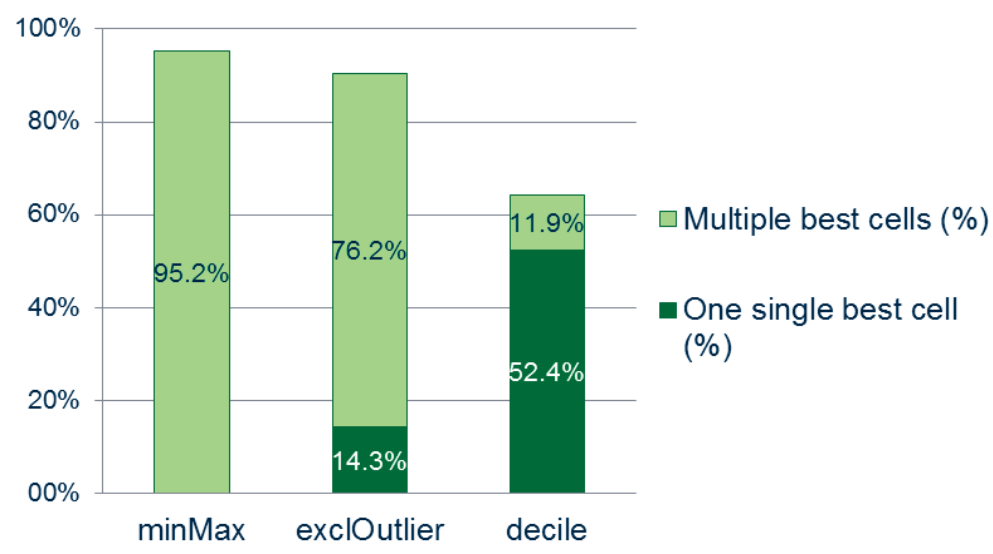

Figure 3. Matching result: One and multiple best matching cell(s)

Table 2. Multiple best matching cells

\begin{tabular}{|l|l|l|l|}
\hline Cell Pattern & minMax & exclOutlier & decile \\
\hline Average no. of best matching cells & 6.4 & 3.5 & 1.2 \\
\hline
\end{tabular}

Best results of more than $95 \%$ accurate matching were achieved by the minimum to maximum pattern accepting more than one cell to be activated. Further studies are planned to investigate additional matching patterns and the impact of multiple switched off cells on the stability of the algorithms.

\section{ACKNOWLEDGMENTS}

We would like to thank Siegfried Klein and Edgar Kuehn from Alcatel-Lucent Bell Labs, Germany for their support with the simulator libraries.

\section{REFERENCES}

[1] 3GPP Evolved Universal Terrestrial Radio Access Network (E-UTRAN). 2011. Self-configuring and self-optimizing network (SON) use cases and solutions (release 9). Technical Specification. TR 36.902, V9.3.1.
[2] 3GPP Telecommunication Management. 2010. Study on Energy Saving Management (ESM) (release 10). Technical Specification. TS 32.826, V10.0.0.

[3] Biczók, G., and Fehske, A. 2011. Economic and Ecological Impact of ICT. EARTH deliverable D2.1, V2.0.

[4] Roth-Mandutz E. and Mitschele-Thiel, A. 2013. LTE Energy Saving SON Using Fingerprinting for Identification of Cells to be Activated. In Future Network and Mobile Summit (FutureNetworkSummit (Lisbon, Portugal, July 03-05, 2013), 1-8.

[5] Tukey, J. W. 1977. Exploratory Data Analysis. AddisonWesley.

[6] Yang, Z. and Jian, L. and Wu, C. and Liu, Y. 2013. Beyond triangle inequality: sifting noisy and outlier distance measurements for localization. ACM Transactions on Sensor Networks (TOSN), 9, 2, 26 